\title{
UNIVERSAL APPROXIMATION THEOREM FOR DIRICHLET SERIES
}

\author{
O. DEMANZE AND A. MOUZE
}

Received 14 September 2005; Revised 11 May 2006; Accepted 30 May 2006

The paper deals with an extension theorem by Costakis and Vlachou on simultaneous approximation for holomorphic function to the setting of Dirichlet series, which are absolutely convergent in the right half of the complex plane. The derivation operator used in the analytic case is substituted by a weighted backward shift operator in the Dirichlet case. We show the similarities and extensions in comparing both results. Several density results are proved that finally lead to the main theorem on simultaneous approximation.

Copyright (c) 2006 Hindawi Publishing Corporation. All rights reserved.

\section{Introduction}

Let $f(s)=\sum_{n \geq 1} a_{n} n^{-s}$ be a Dirichlet series and let $\sigma_{a}(f)$ be its abscissa of absolute convergence, defined by

$$
\sigma_{a}(f)=\inf \left\{\sigma \in \mathbb{R} ; \sum_{n \geq 1}\left|a_{n}\right| n^{-\sigma} \text { converges }\right\} .
$$

We denote $\left\|\sum_{n \geq 1} a_{n} n^{-s}\right\|_{\sigma}=\sum_{n \geq 1}\left|a_{n}\right| n^{-\sigma} \in[0,+\infty]$ for all $\sigma \in \mathbb{R}$. If $f$ is given by a finite sum of the previous type, then we say that $f$ is a Dirichlet polynomial. Let $\mathbb{C}_{+}$be the halfplane of complex numbers with strictly positive real part. We denote by $\mathscr{D}_{a}\left(\mathbb{C}_{+}\right)$the set of Dirichlet series which are absolutely convergent on $\mathbb{C}_{+} \cdot$ This space $\mathscr{D}_{a}\left(\mathbb{C}_{+}\right)$, endowed with the topology given by the family of seminorms $\|\cdot\|_{\sigma}$, is a Fréchet space. In the following, we fix $\tilde{\sigma}=\left(\sigma_{k}\right)_{k \geq 0}$ to be a strictly decreasing sequence of real numbers which converges to 0 . Then, the distance associated to the Fréchet space is defined by

$$
d_{\tilde{\sigma}}(f, g)=\sum_{n \geq 0} \frac{1}{2^{n}} \frac{\|f-g\|_{\sigma_{n}}}{1+\|f-g\|_{\sigma_{n}}}, \quad f \text { and } g \text { are in } \mathscr{D}_{a}\left(\mathbb{C}_{+}\right) \text {. }
$$

The purpose of this work is to obtain, for Dirichlet series in $\mathscr{D}_{a}\left(\mathbb{C}_{+}\right)$, a simultaneous approximation theorem analogous with the one proved by Costakis and Vlachou for holomorphic functions [5]; see also [6]. Let $\Omega$ be a Jordan domain included in $\mathbb{C}$ and let 
$\xi \in \Omega$ be a fixed complex number. We denote by ${ }^{W}$ the set of holomorphic functions $f$ on $\Omega(f \in H(\Omega))$ such that the following holds. For every choice of compact sets $K$ and $L$, with $L \subset \Omega$ and $K \subset \mathbb{C} \backslash \bar{\Omega}$, with connected complement and for every functions $\phi$ and $\psi$, continuous in $K$ and $L$, respectively, analytic in the interiors $\stackrel{\circ}{K}$ and $\stackrel{\circ}{L}$, the following hold.

There exists a sequence $\left(\lambda_{n}\right)_{n \geq 0}$ of nonnegative integers such that

$$
\begin{aligned}
\sup _{z \in K} \mid & \sum_{j=0}^{\lambda_{n}} \frac{f^{(j)}(\xi)}{j !}(z-\xi)^{j}-\phi(z) \mid \underset{n \rightarrow+\infty}{\longrightarrow} 0, \\
& \sup _{z \in L}\left|f^{\left(\lambda_{n}\right)}(z)-\psi(z)\right| \underset{n \rightarrow+\infty}{\longrightarrow} 0 .
\end{aligned}
$$

THEOREM 1.1 [5]. The intersection of $\mathcal{W}$ with the set of univalent functions and constant ones (denote by $\mathscr{B}$ ) is $G_{\delta}$-dense in $\mathscr{B}$.

This universal theorem can be proved using category type of arguments and applying an approximation theorem due to Mergelyan for analytic functions. The arguments are now well known. We can mention $[4,6,7]$ for similar proofs. The idea of [6] is the following. If we have some approximation properties (realized by sequence of natural numbers) which hold on $G_{\delta}$ and dense subsets of a complete metric space, then by Baire's theorem, the intersection of these sets is also $G_{\delta}$ and dense. Therefore, there is an object realizing all those approximations (but with different sequences of indices for each one). Now if we repeat simultaneously the proofs of these generic approximation properties in some cases, a miracle happens. We realize all of them generically with the same choice of indices.

For the set of Dirichlet series $\mathscr{D}_{a}\left(\mathbb{C}_{+}\right)$, a technical version of Mergelyan's theorem has recently been proved by Bayart [3]. This result uses the notion of compact sets $K \subset \mathbb{C I}$ $\mathbb{C}_{+}$admissible for Dirichlet series (see Definition 1.4). For every Dirichlet series $f(z)=$ $\sum_{k=1}^{+\infty} f_{k} k^{-z}$, we denote by $S_{n}(f), n \in \mathbb{N}^{*}$, the partial sum

$$
S_{n}(f)(z)=\sum_{k=1}^{n} f_{k} k^{-z}, \quad z \in \mathbb{C} .
$$

Definition 1.2. Let $\omega=\left(\omega_{n}\right)_{n \in \mathbb{N}^{*}}$ be a sequence of strictly positive real numbers. Denote by $B_{\omega}$ the backward weighted shift defined by

$$
B_{\omega}(1)=0, \quad B_{\omega}\left(n^{-s}\right)=\frac{1}{\omega_{n-1}}(n-1)^{-s}, \quad \forall n \geq 2 .
$$

The purpose of this paper is to give an analogy for Dirichlet series to the results of [5]. The study of derivative for analytic function can be seen as the study of a weighted backward shift (with the canonical basis)

$$
D\left(z^{n}\right)=n z^{n-1}, \quad \forall n \geq 2 .
$$


For this paper, we just consider weights $\omega=\left(\omega_{n}\right)_{n \in \mathbb{N} *}$ satisfying

$\left(H_{1}\right)$

$$
\forall \alpha \geq 0, \quad \omega_{1} \cdots \omega_{m} m^{\alpha} \longrightarrow 0, \quad \text { as } m \longrightarrow+\infty
$$

$\left(H_{2}\right)$

$$
\forall \alpha>0, \quad \text { the sequence }\left(\frac{1}{j^{\alpha} \omega_{j}}\right)_{j \in \mathbb{N}^{*}} \text { is bounded. }
$$

We denote by $M_{\alpha}$ the supremum of the bounded sequence $\left(1 / j^{\alpha} \omega_{j}\right)_{j \in \mathbb{N}^{*}}$. These conditions mean that the infinite product converges to zero but not too quickly. For example, we can choose

$$
\forall k \geq 2, \quad \omega_{k}=\frac{C}{[\log (k)]^{\beta}}, \quad C \in \mathbb{R}_{+}^{*}, \beta>0 .
$$

Simpler examples can be chosen, by taking $\omega_{k}=1 / \lambda$ with $\lambda>1$. Moreover, we can note that the second condition implies that the $B_{\omega}$ are continuous operators on the Fréchet space $\mathscr{D}_{a}\left(\mathbb{C}_{+}\right)$. We have for $f(s)=\sum_{n \geq 1} a_{n} n^{-s} \in \mathscr{D}_{a}\left(\mathbb{C}_{+}\right)$,

$$
\begin{aligned}
\left\|B_{\omega}(f)\right\|_{\alpha_{n}} & =\sum_{j \geq 1}\left|a_{j+1}\right| \frac{j^{-\alpha_{n}}}{\omega_{j}}=\sum_{j \geq 1}\left|a_{j+1}\right|\left(\frac{j}{j+1}\right)^{-\alpha_{n+1}} \frac{(j+1)^{-\alpha_{n+1}}}{j^{\alpha_{n}-\alpha_{n+1}} \omega_{j}} \\
& \leq 2^{\alpha_{n+1}} M_{\alpha_{n}-\alpha_{n+1}}\|f\|_{\alpha_{n+1}} .
\end{aligned}
$$

Therefore, we obtain the following result, as a direct consequence of the main Theorem 4.3, which generalizes Bayart [3, Theorem 6]. This result is Corollary 4.4 and states the following.

Corollary 1.3. Let $f \in \mathscr{D}_{a}\left(\mathbb{C}_{+}\right)$be a Dirichlet series. Then, for every $\varepsilon>0$, there exists $h \in \mathscr{D}_{a}\left(\mathbb{C}_{+}\right)$satisfying the following. For every $\varphi \in \mathscr{D}_{a}\left(\mathbb{C}_{+}\right)$, every admissible compact set $K$ in $\overline{\mathbb{C}_{-}}$, and every function $\psi: K \rightarrow \mathbb{C}$ continuous on $K$ and holomorphic in $\stackrel{\circ}{K}$, there exists a sequence $\left(\lambda_{n}\right)_{n \geq 0}$ in $\mathbb{N}^{*}$ such that the following hold:

$$
\begin{gathered}
d_{\tilde{\sigma}}(f, h)<\varepsilon, \\
\sup _{z \in K}\left|S_{\lambda_{n}}(h)(z)-\psi(z)\right| \longrightarrow 0, \quad \text { as } n \longrightarrow+\infty, \\
B_{\omega}^{\lambda_{n}}(h) \longrightarrow \varphi, \quad \text { as } n \longrightarrow+\infty, \text { in } \mathscr{D}_{a}\left(\mathbb{C}_{+}\right),
\end{gathered}
$$

where $B_{\omega}^{\lambda_{n}}$ is the $\lambda_{n}$ th iteration of the weighted backward shift.

The method of the proof is analogous to the one of the main theorem in [5]. We use principally the version of Mergelyan's theorem for Dirichlet series given by Bayart [3]. The main tool of approximation by Dirichlet series is a technical lemma from Bagchi [1] which needs the notion of admissible compact set. 
Definition 1.4. Let $K$ be a compact set included in $\mathbb{C}$. This set is admissible for Dirichlet series if $\mathbb{C} \backslash K$ is connected, and if the following representation can be obtained: $K=$ $K_{1} \cup \cdots \cup K_{d}$, where the $K_{i}$ should be contained in disconnected strips $S_{i}=\left\{z \in \mathbb{C} ; a_{i} \leq\right.$ $\left.\mathfrak{R}(z) \leq b_{i}\right\}$ breadth strictly less than $1 / 2$, that is, $b_{i}-a_{i}<1 / 2$.

We denote by $\mathbb{C}_{-}$the left half-plane $\{s \in \mathbb{C} ; \mathfrak{R}(s)<0\}$. We can now express the version of Mergelyan's theorem for Dirichlet series included in $\mathscr{D}_{a}\left(\mathbb{C}_{+}\right)$.

Theorem 1.5 [3]. Let $K \subset \overline{\mathbb{C}_{-}}$be an admissible compact set for Dirichlet series, let $f$ be a Dirichlet series in $\mathscr{D}_{a}\left(\mathbb{C}_{+}\right)$, and let $g$ be a continuous function on $K$ which is analytic in $\stackrel{\circ}{K}$. For every pair of fixed positive real numbers $\sigma$ and $\varepsilon$, there exists a Dirichlet polynomial $h$ in $\mathscr{D}_{a}\left(\mathbb{C}_{+}\right)$such that

$$
\sup _{z \in K}|h(z)-g(z)|<\varepsilon, \quad\|h-f\|_{\sigma}<\varepsilon .
$$

Definition 1.6. Denote by $\mathscr{W}_{d}(\omega)$ the set of all Dirichlet series $h \in \mathscr{D}_{a}\left(\mathbb{C}_{+}\right)$satisfying the following: for every admissible compact set $K$ in $\overline{\mathbb{C}_{-}}$, for every $f \in \mathscr{D}_{a}\left(\mathbb{C}_{+}\right)$, and for every function $g: K \rightarrow \mathbb{C}$ continuous on $K$ and holomorphic in $\stackrel{\circ}{K}$, there exists a sequence of integers $\left(\lambda_{n}\right)_{n \geq 0}$ such that the following hold:

$$
\begin{gathered}
\sup _{z \in K}\left|S_{\lambda_{n}}(h)(z)-g(z)\right| \underset{n \rightarrow+\infty}{\longrightarrow} 0, \\
B_{\omega}^{\lambda_{n}}(h) \underset{n \rightarrow+\infty}{\longrightarrow} f \quad \text { in } \mathscr{D}_{a}\left(\mathbb{C}_{+}\right) .
\end{gathered}
$$

In a first step, we give relations between the set $\mathcal{W}_{d}(\omega)$ and subsets of $\mathscr{D}_{a}\left(\mathbb{C}_{+}\right)$realizing analogous estimations with Dirichlet polynomials. On the other hand, we prove that these subsets are open and that their union is dense in the Fréchet space studied. We conclude using category-type arguments (see also $[4,5,7]$ ). Other recent developments related to universal Dirichlet series have been obtained in the same way in [8].

\section{The sets $\mathbb{O}_{\sigma_{k}}\left(\mathbb{C}_{+}, \rho, j, s, n\right)$}

Definition 2.1. The family of compact sets is defined as

$$
K\left(a_{i} ; b_{i} ; n\right)=\left\{z \in \mathbb{C} \text { such that } a_{i} \leq \mathfrak{R}(z) \leq b_{i} \text { et } \mathfrak{I}(z) \in[-n ; n]\right\},
$$

where $\left.a_{i} \in \mathbb{Q}, b_{i} \in \mathbb{Q} \cap\right] a_{i} ; a_{i}+1 / 2\left[, n \in \mathbb{N}^{*}\right.$, and $b_{i} \leq 0$. For all $d \in \mathbb{N}^{*}$, let $K\left(a_{1} ; b_{1} ; \ldots ; a_{d}\right.$; $\left.b_{d}\right)$ be the disjoint union of the sets $K\left(a_{i} ; b_{i} ; n_{i}\right)$ for $i \in\{1, \ldots, d\}$. The family of compact sets $K\left(a_{1} ; b_{1} ; \ldots ; a_{d} ; b_{d}\right)$ is denumerable. This family can be denoted by the sequence $\left(K_{\rho}\right)_{\rho \geq 0}$.

It is obvious that the sets $K_{\rho}$ are well admissible for Dirichlet series and are contained in $\overline{\mathbb{C}_{-}}$.

Proposition 2.2. For each admissible (for Dirichlet series) compact set $K \subset \overline{\mathbb{C}_{-}}$, there exists a nonnegative integer $\rho_{0}$ such that the following inclusion holds:

$$
K \subset K_{\rho_{0}} .
$$


Proof. We just have to prove this property for a compact set $K$ included in only one strip $S=\{z \in \mathbb{C} / a \leq \mathfrak{R}(z) \leq b\}$ with $b-a<1 / 2$. Using the notations above, if $(a, b) \in \mathbb{Q}^{2}$, we just have to choose $n \geq \sup \{|z| / z \in K\}$. Else, we take $a^{\prime}<a$ and $b^{\prime}>b$ such that $\left(a^{\prime}, b^{\prime}\right) \in$ $\mathbb{Q}^{2}$ and $b^{\prime}-a^{\prime}<1 / 2$. Hence, with the same choice for $n$, we obtain the desired relation $K \subset K\left(a^{\prime} ; b^{\prime} ; n\right)$.

In the following, we denote the set of Dirichlet polynomials with coefficients in $\mathbb{Q}+i \mathbb{Q}$ by the sequence $\left(f_{j}\right)_{j \in \mathbb{N}}$.

Lemma 2.3. The family of Dirichlet polynomials $\left(f_{j}\right)_{j \in \mathbb{N}}$ is a dense set for the topology of the Fréchet space $\mathscr{D}_{a}\left(\mathbb{C}_{+}\right)$.

Proof. Let $g=\sum_{k=1}^{+\infty} \alpha_{k} k^{-s}$ be a Dirichlet series $\mathscr{D}_{a}\left(\mathbb{C}_{+}\right)$. We define the sequence $\left(f_{j_{n}}\right)_{n \geq 0}$ by

$$
f_{j_{n}}=\sum_{k=1}^{n} \beta_{k, n} k^{-s}, \quad\left|\alpha_{k}-\beta_{k, n}\right| \leq \frac{1}{2^{k+n}}, \quad \beta_{k, n} \in \mathbb{Q}+i \mathbb{Q} .
$$

Let $\sigma$ be a fixed strictly positive real number and let $\varepsilon$ be a strictly positive real number too. Clearly, there exists a positive integer $N$ such that we have $\sum_{k=N+1}^{+\infty}\left|\alpha_{k}\right| k^{-\sigma}<\varepsilon$. Therefore, we obtain the following inequality:

$$
\left\|g-f_{j_{n}}\right\|_{\sigma}=\sum_{k=1}^{N}\left|\alpha_{k}-\beta_{k, n}\right| k^{-\sigma}+\sum_{k=N+1}^{+\infty}\left|\alpha_{k}\right| k^{-\sigma}<\sum_{k=1}^{N} \frac{1}{2^{k+n}}+\varepsilon<\frac{1}{2^{n}}+\varepsilon .
$$

The conclusion is obvious.

Remark 2.4. We obviously have the density of the family $\left(f_{j}\right)_{j \in \mathbb{N}}$ for the topology of the uniform (on every compact set) convergence.

Definition 2.5. Let $\sigma>0$. According to the preceding definitions, for all positive integers $\rho, j, n, s$, the set $O_{\sigma}\left(\mathbb{C}_{+}, \rho, j, s, n\right) \subset \mathscr{D}_{a}\left(\mathbb{C}_{+}\right)$is defined by

$\widehat{O}_{\sigma}\left(\mathbb{C}_{+}, \rho, j, s, n\right)=\left\{g \in \mathscr{D}_{a}\left(\mathbb{C}_{+}\right)\right.$such that $\left.\sup _{z \in K_{\rho}}\left|S_{n}(g)(z)-f_{j}(z)\right|<\frac{1}{s},\left\|B_{\omega}^{n}(g)-f_{j}\right\|_{\sigma}<\frac{1}{s}\right\}$.

With these sets, we have a complete representation of $\mathcal{W}_{d}(\omega)$.

Lemma 2.6. The following equality holds:

$$
\mathcal{W}_{d}(\omega)=\bigcap_{\rho=1}^{+\infty} \bigcap_{k=1}^{+\infty} \bigcap_{j=0}^{+\infty} \bigcap_{s=1}^{+\infty} \bigcup_{n=1}^{+\infty} \mathcal{O}_{\sigma_{k}}\left(\mathbb{C}_{+}, \rho, j, s, n\right)
$$

Proof. Let $g$ be a Dirichlet series belonging to the right-hand side set. Let $K \subset \overline{\mathbb{C}_{-}}$be an admissible compact set for Dirichlet series and $\Phi: K \rightarrow \mathbb{C}$ be continuous function on $K$ and analytic in $\stackrel{\circ}{K}$, and let $h$ be in $\mathscr{D}_{a}\left(\mathbb{C}_{+}\right)$. For all $\varepsilon>0$ and $\sigma>0$, we just have to find an 
6 Universal approximation theorem for Dirichlet series

integer $n_{0} \in \mathbb{N}$ such that we have

$$
\sup _{z \in K}\left|S_{n_{0}}(g)(z)-\Phi(z)\right|<\varepsilon, \quad\left\|B_{\omega}^{n_{0}}(g)-h\right\|_{\sigma}<\varepsilon .
$$

Using Bayart's Theorem 1.5, there exists a Dirichlet polynomial $p$ satisfying the two inequalities

$$
\sup _{z \in K}|p(z)-\Phi(z)|<\frac{\varepsilon}{2}, \quad\|p-h\|_{\sigma}<\frac{\varepsilon}{2}
$$

With no loss of generality, by Lemma 2.3, we may assume that its coefficients are in $\mathbb{Q}+$ $i \mathbb{Q}$. As a consequence, there exists an integer $j_{0} \in \mathbb{N}$ such that $p=f_{j_{0}}$. We let $s_{0}$ and $\rho_{0}$ be nonnegative integers satisfying $2 \leq s_{0} \varepsilon$ and $K \subset K_{\rho_{0}}$. Moreover there exists $n_{0}$ such that

$$
\sup _{z \in K}\left|S_{n_{0}}(g)(z)-p(z)\right|<\frac{1}{s_{0}}, \quad\left\|B_{\omega}^{n_{0}}(g)-p\right\|_{\sigma}<\frac{1}{s_{0}} .
$$

The last four inequalities allow us to conclude that $g$ belongs to $\mathcal{W}_{d}(\omega)$. The inverse inclusion is obvious.

\section{The sets $O_{\sigma_{k}}\left(\mathbb{C}_{+}, \rho, j, s, n\right)$ are open}

In this section, we prove that each set $\bigcup_{n \geq 1} \widetilde{O}_{\sigma}\left(\mathbb{C}_{+}, \rho, j, s, n\right)$ is open according to the Fréchet topology defined by the seminorms $\left(\|\cdot\|_{\beta}\right)_{\beta}$ on $\mathscr{D}_{a}\left(\mathbb{C}_{+}\right)$. We divide the proof into two parts.

Proposition 3.1. For all $j, s, n$, and $\rho$ positive integers, the subsets $\Gamma(\rho, j, s, n)$ of $\mathscr{D}_{a}\left(\mathbb{C}_{+}\right)$ are open in the Fréchet space $\mathscr{D}_{a}\left(\mathbb{C}_{+}\right)$, where

$$
\Gamma(\rho, j, s, n)=\left\{g \in \mathscr{D}_{a}\left(\mathbb{C}_{+}\right) \text {such that } \sup _{z \in K_{\rho}}\left|S_{n}(g)(z)-f_{j}(z)\right|<\frac{1}{s}\right\} .
$$

Proof. We denote by $M$ the minimum of the real parts of the complex numbers included in $K_{\rho}$. Let $f$ be a Dirichlet series in $\Gamma(\rho, j, s, n)$, which means that we have

$$
\sup _{z \in K_{\rho}}\left|S_{n}(f)(z)-f_{j}(z)\right|<\frac{1}{s} .
$$

Let $\varepsilon_{1}$ be the following strictly positive real number:

$$
\varepsilon_{1}=\frac{1 / s-\sup _{z \in K_{\rho}}\left|S_{n}(f)(z)-f_{j}(z)\right|}{\sum_{r=1}^{n} r^{\sigma_{0}} r^{-M}}>0 .
$$

Define $a=\varepsilon_{1} /\left(1+\varepsilon_{1}\right)$ and let $g$ be a Dirichlet series satisfying $d_{\tilde{\sigma}}(g, f)<a$. In particular, we obtain

$$
\frac{\|f-g\|_{\sigma_{0}}}{1+\|f-g\|_{\sigma_{0}}}<a \text { which means }\|f-g\|_{\sigma_{0}}<\varepsilon_{1} .
$$


Now, we can overestimate $\left|S_{n}(g)(z)-f_{j}(z)\right|$. One has

$$
\left|S_{n}(g)(z)-f_{j}(z)\right| \leq\left|S_{n}(g-f)(z)\right|+\left|S_{n}(f)(z)-f_{j}(z)\right| .
$$

Afterwards, if we denote $S_{n}(g-f)(z)=\sum_{r=1}^{n} b_{r} r^{-z}$, using (3.4), we have the following inequality:

$$
\left|b_{r}\right| \leq r^{\sigma_{0}} \sum_{r=1}^{n}\left|b_{r}\right| r^{-\sigma_{0}}<r^{\sigma_{0}} \varepsilon_{1}
$$

Consequently, one has

$$
\sup _{z \in K_{\rho}}\left|S_{n}(g-f)(z)\right|<\sum_{r=1}^{n} \varepsilon_{1} r^{\sigma_{0}-M} .
$$

Hence, we obtain the strict inequality

$$
\sup _{z \in K_{\rho}}\left|S_{n}(g)(z)-f_{j}(z)\right|<\varepsilon_{1} \sum_{r=1}^{n} r^{\sigma_{0}-M}+\sup _{z \in K_{\rho}}\left|S_{n}(f)(z)-f_{j}(z)\right|=\frac{1}{s} .
$$

Therefore, the set $\Gamma(\rho, j, s, n)$ is open in the Fréchet topology.

Proposition 3.2. For all positive integers $j, s, n$, and $\rho$, the subsets $\Delta_{\sigma_{k}}(\rho, j, s, n)$ are open in the Fréchet space $\mathscr{D}_{a}\left(\mathbb{C}_{+}\right)$, where

$$
\Delta_{\sigma_{k}}(\rho, j, s, n)=\left\{g \in \mathscr{D}_{a}\left(\mathbb{C}_{+}\right) \text {such that }\left\|B_{\omega}^{n}(g)-f_{j}\right\|_{\sigma_{k}}<\frac{1}{s}\right\} .
$$

Proof. We begin this proof as the preceding one. Let $f$ be a Dirichlet series included in $\Delta_{\sigma_{k}}(\rho, j, s, n)$. Hence

$$
\left\|B_{\omega}^{n}(f)-f_{j}\right\|_{\sigma_{k}}<\frac{1}{s}
$$

Take $\varepsilon_{2}$ to be a strictly positive real number satisfying

$$
\varepsilon_{2} \leq \frac{1 / s-\left\|B_{\omega}^{n}(f)-f_{j}\right\|_{\sigma_{k}}}{A_{n, k}}, \quad \text { with } A_{n, k}=\sup _{j \geq 1}\left(\frac{j}{j+n}\right)^{-\sigma_{k+1}} \sup _{j} \frac{j^{\sigma_{k+1}-\sigma_{k}}}{\omega_{j} \cdots \omega_{j+n-1}} .
$$

Note that $A_{n, k}$ is well defined because $j /(j+n) \rightarrow 1$ and the second supremum is well defined by $\left(H_{2}\right)$. Now, define $a=\varepsilon_{2} / 2^{k+1}\left(1+\varepsilon_{2}\right)$ and let $g$ be a Dirichlet series satisfying $d_{\tilde{\sigma}}(g, f)<a$. In particular, we obtain $\|f-g\|_{\sigma_{k+1}} /\left(1+\|f-g\|_{\sigma_{k+1}}\right)<2^{k+1} a$ so $\| f-$ $g \|_{\sigma_{k+1}}<\varepsilon_{2}$. Now we can estimate $\left\|B_{\omega}^{n}(g)-f_{j}\right\|_{\sigma_{k}}$. It follows that

$$
\left\|B_{\omega}^{n}(g)-f_{j}\right\|_{\sigma_{k}} \leq\left\|B_{\omega}^{n}(g-f)\right\|_{\sigma_{k}}+\left\|B_{\omega}^{n}(f)-f_{j}\right\|_{\sigma_{k}} .
$$

Using the notation $(g-f)(z)=\sum_{r=1}^{+\infty} b_{r} r^{-z}$, we also have

$$
\left\|B_{\omega}^{n}(g-f)\right\|_{\sigma_{k}}=\sum_{j=1}^{+\infty} \frac{\left|b_{j+n}\right|}{\omega_{j} \cdots \omega_{j+n-1}} j^{-\sigma_{k}} \leq A_{n, k}\|g-f\|_{\sigma_{k+1}} .
$$


As a consequence, we obtain the following inequality:

$$
\begin{aligned}
\left\|B_{\omega}^{n}(g)-f_{j}\right\|_{\sigma_{k}} & \leq\left\|B_{\omega}^{n}(g-f)\right\|_{\sigma_{k}}+\left\|B_{\omega}^{n}(f)-f_{j}\right\|_{\sigma_{k}} \\
& \leq A_{n, k}\|g-f\|_{\sigma_{k+1}}+\left\|B_{\omega}^{n}(f)-f_{j}\right\|_{\sigma_{k}} \\
& <A_{n, k} \varepsilon_{2}+\left\|B_{\omega}^{n}(f)-f_{j}\right\|_{\sigma_{k}} \leq \frac{1}{s} .
\end{aligned}
$$

Therefore, there exists an open ball, with $f$ as central point, which is included into the set $\Delta_{\sigma_{k}}(\rho, j, s, n)$.

Corollary 3.3. For all positive integers $j, s, n, \rho$ and $k$, the set $O_{\sigma_{k}}\left(\mathbb{C}_{+}, \rho, j, s, n\right)$ is open in the Fréchet space $\mathscr{D}_{a}\left(\mathbb{C}_{+}\right)$.

Proof. We just have to remark that the set $\widetilde{O}_{\sigma_{k}}\left(\mathbb{C}_{+}, \rho, j, s, n\right)$ is the intersection of $\Delta_{\sigma_{k}}(\rho, j, s$, $n)$ with $\Gamma(\rho, j, s, n)$ which are open sets due to Propositions 3.1 and 3.2.

\section{Main results}

Proposition 4.1. Let $g$ be a Dirichlet polynomial denoted by $g(z)=\sum_{k=2}^{A} g_{k} k^{-z}$. Then, there exists a sequence of Dirichlet polynomials, $\left(p_{m}\right)_{m \geq 0}$, such that $\left\|p_{m}\right\|_{\sigma} \rightarrow 0$, as $m \rightarrow+\infty$, for all $\sigma \in \mathbb{R}$, and

$$
\forall z \in \mathbb{C}, \forall m \in \mathbb{N}, \quad B_{\omega}^{m}\left(p_{m}\right)(z)=g(z) .
$$

Proof. Using the notations of the proposition $g(z)=\sum_{k=1}^{A} g_{k} k^{-z}$, we consider the Dirichlet polynomials $p_{m}(z)=\sum_{k=1}^{A} g_{k} \omega_{k} \omega_{k+1} \cdots \omega_{k+m-1}(k+m)^{-z}$. With an easy computation, we obtain the desired equalities. Moreover, we have for all $\sigma \in \mathbb{R}$,

$$
\begin{gathered}
\text { case } \sigma \geq 0:\left\|p_{m}\right\|_{\sigma} \leq \sum_{k=1}^{A}\left|g_{k}\right| \omega_{k} \omega_{k+1} \cdots \omega_{k+m-1} k^{-\sigma}, \\
\text { case } \sigma<0:\left\|p_{m}\right\|_{\sigma} \leq \sum_{k=1}^{A}\left|g_{k}\right| \omega_{k} \omega_{k+1} \cdots \omega_{k+m-1}(k+m)^{-\sigma} .
\end{gathered}
$$

Using $\left(H_{1}\right)$, we obtain the result.

Note that the sequence $\left(p_{m}\right)_{m \geq 0}$ constructed in the previous proposition converges also uniformly (on each compact set) to 0 .

We possess all the arguments to state and to prove the main theorem of this work.

Theorem 4.2. The sets $\bigcup_{n=0}^{\infty} \mathscr{O}_{\sigma_{k}}\left(\mathbb{C}_{+}, \rho, j, s, n\right)$ are dense subsets of the Fréchet space $\mathscr{D}_{a}\left(\mathbb{C}_{+}\right)$.

Proof. Let $d \in \mathscr{D}_{a}\left(\mathbb{C}_{+}\right)$be written $d(z)=\sum_{j=1}^{\infty} a_{j} j^{-z}$. We want to obtain a sequence of Dirichlet polynomials in $\bigcup_{n=0}^{\infty} \widehat{O}_{\sigma_{k}}\left(\mathbb{C}_{+}, \rho, j, s, n\right)$ which converges to $d$. Fix $\left.\varepsilon \in\right] 0 ; 1 / 2 s$ [ and $\beta \in \tilde{\sigma}$. We denote by $d_{r}(z)$ the partial sum $\sum_{j=1}^{r} a_{j} j^{-z}$ and we choose $r$ such that $\| d-$ $d_{r} \|_{\beta}<\varepsilon$. Now, we approximate the Dirichlet polynomial $d_{r}$ with an element in $\bigcup_{n=0}^{\infty} \widehat{O}_{\sigma_{k}}\left(\mathbb{C}_{+}\right.$, $\rho, j, s, n)$. According to Theorem 1.5, for all $m>0$, there exists a Dirichlet polynomial $p_{m}$ 
satisfying the following inequalities:

$$
\sup _{z \in K_{\rho}}\left|p_{m}(z)-f_{j}(z)\right|<\frac{1}{m}, \quad\left\|p_{m}-d_{r}\right\|_{\beta}<\frac{1}{m} .
$$

Due to Proposition 4.1, there exists a sequence of Dirichlet polynomials, $\left(q_{m}\right)_{m \geq 0}$, such that $\left\|q_{m}\right\|_{\sigma} \rightarrow 0$, as $m \rightarrow+\infty$, for all $\sigma \in \mathbb{R}$ and $B_{\omega}^{m}\left(q_{m}\right)(z)=f_{j}(z)$. With the notation $f_{j}(z)=\sum_{l=1}^{N_{j}} \alpha_{l, j} l^{-z}$, we know that $q_{m}$ can be described by

$$
q_{m}(z)=\sum_{l=1}^{N_{j}} \alpha_{l, j} \omega_{l} \omega_{l+1} \cdots \omega_{l+m-1}(l+m)^{-z} .
$$

With this representation, we obtain $S_{m}\left(q_{m}\right)(z) \equiv 0$. From (4.3), there exists an integer $m_{0}$ such that we have $\sup _{z \in K_{\rho}}\left|p_{m_{0}}(z)-f_{j}(z)\right|<1 / s$. We denote $p_{m_{0}}(z)=\sum_{l=1}^{N_{m_{0}}} b_{l, m_{0}} l^{-z}$ and take $m_{1}>\max \left(m_{0} ; N_{m_{0}}\right)$ such that

$$
\left\|q_{m_{1}}\right\|_{\beta}=\sum_{l=1}^{N_{j}}\left|\alpha_{l, j}\right| \omega_{l} \omega_{l+1} \cdots \omega_{l+m_{1}-1}\left(l+m_{1}\right)^{-\beta}<\frac{1}{2 s} .
$$

As $m_{1}>N_{m_{0}}$, we have the equality $S_{m_{1}}\left(p_{m_{0}}\right)=p_{m_{0}}$. Let $P$ be defined by

$$
P(z)=p_{m_{0}}(z)+q_{m_{1}}(z) .
$$

We deduce from above that $P$ satisfies

$$
\left\|B_{\omega}^{m_{1}}(P)-f_{j}\right\|_{\sigma_{k}}=\left\|B_{\omega}^{m_{1}}\left(p_{m_{0}}\right)\right\|_{\sigma_{k}}=0<\frac{1}{2 s}
$$

because $m_{1}>N_{m_{0}}$. Using $S_{m_{1}}\left(q_{m_{1}}\right) \equiv 0, \sup _{z \in K_{\rho}}\left|S_{m_{1}}(P)(z)-f_{j}(z)\right|=\sup _{z \in K_{\rho}} \mid p_{m_{0}}(z)-$ $f_{j}(z) \mid<1 / s$. Using formula (4.3), we also obtain $\left\|p_{m}-d_{r}\right\|_{\beta}<\varepsilon$. Up to choosing a bigger integer $m_{0}$, we may assume that $\left\|p_{m_{0}}-d_{r}\right\|_{\beta}<\varepsilon$. As we have

$$
\left\|P-d_{r}\right\|_{\beta} \leq\left\|p_{m_{0}}-d_{r}\right\|_{\beta}+\left\|q_{m_{1}}\right\|_{\beta}<2 \varepsilon
$$

we conclude that for a fixed strictly positive real $\beta$, for all $\varepsilon<1 /(2 s)$, there exists a Dirichlet polynomial $P \in \bigcup_{n} \mathcal{O}_{\sigma_{k}}\left(\mathbb{C}_{+}, \rho, j, s, n\right)$ such that $\left\|P-d_{r}\right\|_{\beta}<2 \varepsilon$, which implies the inequality $\|P-d\|_{\beta}<3 \varepsilon$. As it is true for every strictly positive real $\beta$, we obtain the density of $\bigcup_{n=0}^{+\infty} \mathscr{O}_{\sigma_{k}}\left(\mathbb{C}_{+}, \rho, j, s, n\right)$ in $\mathscr{D}_{a}\left(\mathbb{C}_{+}\right)$.

Theorem 4.3. The set $W_{d}(\omega)$ is a $G_{\delta}$ and dense set included in $\mathscr{D}_{a}\left(\mathbb{C}_{+}\right)$.

Proof. Lemma 2.6 implies that $W_{d}(\omega)$ is a countable intersection of dense open sets of $\mathscr{D}_{a}\left(\mathbb{C}_{+}\right)$. Hence, the result is a direct consequence of Baire's theorem.

As a straightforward consequence of Theorem 4.3, we obtain the following explicit result of approximation. 
Corollary 4.4. Let $f \in \mathscr{D}_{a}\left(\mathbb{C}_{+}\right)$be a Dirichlet series. Then, for every $\varepsilon>0$, there exists $h \in \mathscr{D}_{a}\left(\mathbb{C}_{+}\right)$satisfying the following. For every $\varphi \in \mathscr{D}_{a}\left(\mathbb{C}_{+}\right)$, every admissible compact set $K$ in $\overline{\mathbb{C}_{-}}$and every function $\psi: K \rightarrow \mathbb{C}$ continuous on $K$ and holomorphic in $\stackrel{\circ}{K}$, there exists a sequence $\left(\lambda_{n}\right)_{n \geq 0}$ in $\mathbb{N}^{*}$ such that the following hold:

$$
\begin{gathered}
d_{\tilde{\sigma}}(f, h)<\varepsilon, \\
\sup _{z \in K}\left|S_{\lambda_{n}}(h)(z)-\psi(z)\right| \longrightarrow 0, \quad \text { as } n \longrightarrow+\infty, \\
B_{\omega}^{\lambda_{n}}(h) \longrightarrow \varphi, \quad \text { as } n \longrightarrow+\infty, \text { in } \mathscr{D}_{a}\left(\mathbb{C}_{+}\right),
\end{gathered}
$$

where $B_{\omega}^{\lambda_{n}}$ is the $\lambda_{n}$ th iteration of the weighted backward shift.

Remark 4.5. In the article [5], the authors obtain an approximation result using only constant and univalent functions. Here, it is not realistic to obtain such a result because it is well known that a Dirichlet series cannot be univalent on its convergent domain (see, e.g., [2]). The present paper can also be seen as an extension of [6] to universal Dirichlet series. Moreover, Theorem 4.3 is a universal approximation result in the sense of [7]. Note that here the intersection between compact sets and $\overline{\mathbb{C}_{+}}$is not necessary empty.

Remark 4.6. According to a suggestion of Kahane (see [7, Proposition 3.2]), we have the following proposition.

Proposition 4.7. Let $f$ be a Dirichlet series in $\mathscr{D}_{a}\left(\mathbb{C}_{+}\right)$. Then, there exist $g_{1}$ and $g_{2}$ in $W_{d}(\omega)$ such that $f=g_{1}+g_{2}$.

Proof. We use a translation homeomorphism $T_{f}: \mathscr{D}_{a}\left(\mathbb{C}_{+}\right) \rightarrow \mathscr{D}_{a}\left(\mathbb{C}_{+}\right)$defined by $T_{f}(h)=$ $f-h$. As $\mathscr{W}_{d}(\omega)$ is a $G_{\delta}$ and dense set, its image by $T_{f}$ is also a $G_{\delta}$ and dense set. Using Baire's theorem, $\mathscr{W}_{d}(\omega) \cap\left[f-\mathcal{W}_{d}(\omega)\right]$ is no void. Let $g_{1}$ be an element of this intersection. It follows that $g_{1} \in \mathcal{W}_{d}(\omega)$ and $g_{1}=f-g_{2}$ for an element $g_{2} \in \mathcal{W}_{d}(\omega)$.

As for the classical case, there exists a relation between the set $\mathcal{W}_{d}(\omega)$ and the notion of universality in the sense of Menchoff. We do not repeat the proof, it suffices to follow [7, Proposition 3.1].

Proposition 4.8. Let $S=\sum_{n \geq 1} a_{n} n^{-s} \in \mathcal{W}_{d}(\omega)$ and let $g$ and $h$ be two measurable functions from $\mathbb{R}$ into $[-\infty ;+\infty]$. Then there exists a subsequence $S_{k_{m}}$ of the partial sums of $S$ such that

$\mathfrak{R}\left(S_{k_{m}}(i t)\right) \longrightarrow g(t), \quad \mathfrak{I}\left(S_{k_{m}}(i t)\right) \longrightarrow h(t), \quad$ as $m \longrightarrow+\infty$, almost everywhere.

\section{References}

[1] B. Bagchi, A joint universality theorem for Dirichlet L-functions, Mathematische Zeitschrift 181 (1982), no. 3, 319-334.

[2] F. Bayart, Opérateurs de composition sur des espaces de séries de Dirichlet et problèmes d'hypercyclicité simultanée, Ph.D. thesis, University of Lille, Lille, 2002.

[3] _ Topological and algebraic genericity of divergence and universality, Studia Mathematica 167 (2005), no. 2, 161-181. 
[4] G. Costakis, Some remarks on universal functions and Taylor series, Mathematical Proceedings of the Cambridge Philosophical Society 128 (2000), no. 1, 157-175.

[5] G. Costakis and V. Vlachou, A generic result concerning univalent universal functions, Archiv der Mathematik 82 (2004), no. 4, 344-351.

[6] __ Identical approximative sequence for various notions of universality, Journal of Approximation Theory 132 (2005), no. 1, 15-24.

[7] V. Nestoridis, Universal Taylor series, Annales de l'Institut Fourier (Grenoble) 46 (1996), no. 5, 1293-1306.

[8] V. Nestoridis and C. Papadimitropoulos, Abstract theory of universal series and an application to Dirichlet series, Comptes Rendus Mathématique. Académie des Sciences. Paris 341 (2005), no. 9, 539-543.

O. Demanze: Laboratoire de Mathématiques, UMR 8524, Université des Sciences et Technologies de Lille 1 (USTL), Cité Scientifique, 59650 Villeneuve d'Ascq, France E-mail address: olivier.demanze@math.univ-lille1.fr

A. Mouze: Laboratoire de Mathématiques, UMR 8524, Université des Sciences et Technologies de Lille 1 (USTL), Cité Scientifique, 59650 Villeneuve d'Ascq, France E-mail address: augustin.mouze@math.univ-lille1.fr Current address: École Centrale de Lille, Cite Scientifique, BP 48, 59651 Villeneuve d'Ascq Cedex, France 


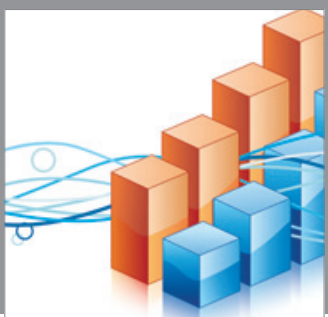

Advances in

Operations Research

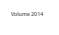

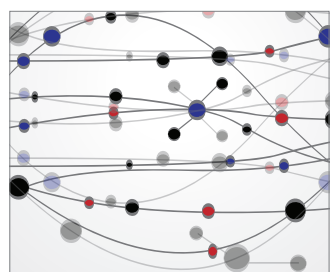

\section{The Scientific} World Journal
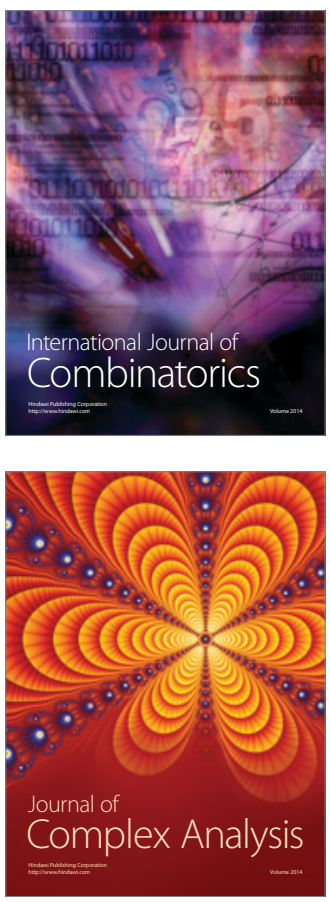

International Journal of

Mathematics and

Mathematical

Sciences
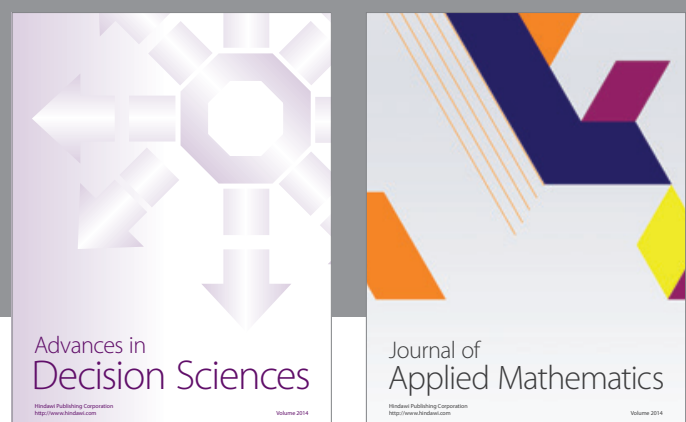

Journal of

Applied Mathematics
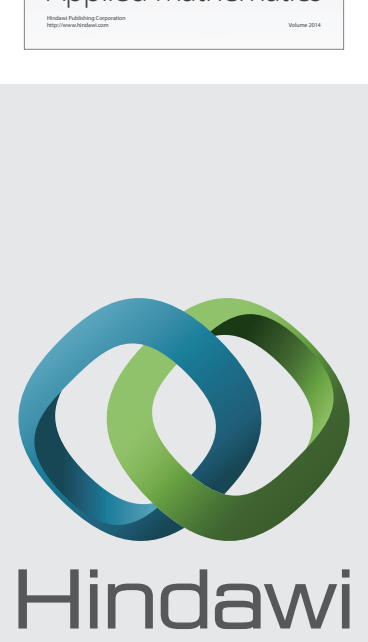

Submit your manuscripts at http://www.hindawi.com
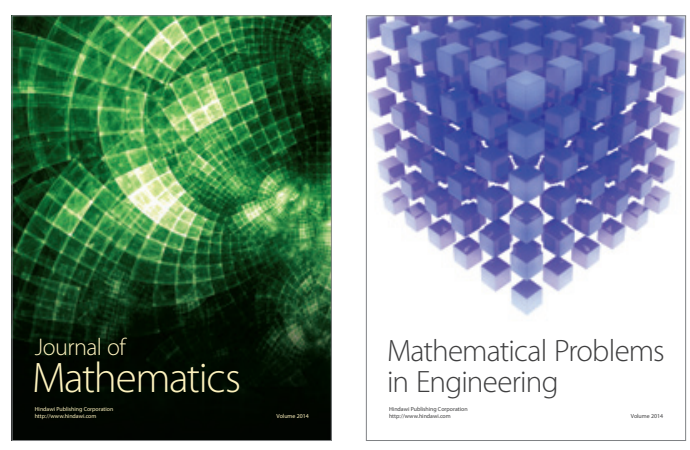

Mathematical Problems in Engineering
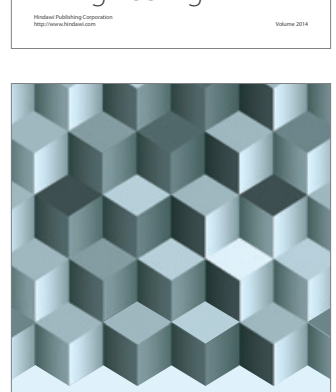

Journal of

Function Spaces
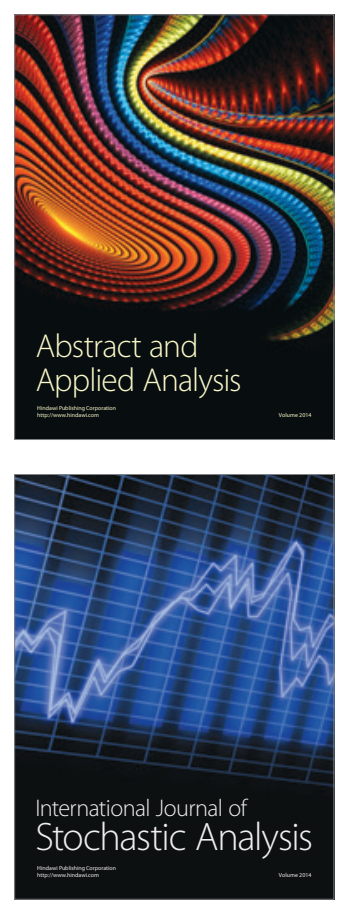

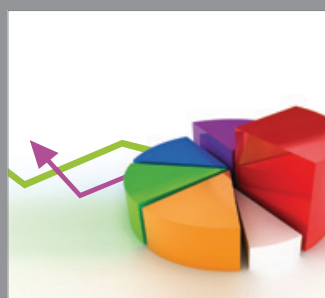

ournal of

Probability and Statistics

Promensencen
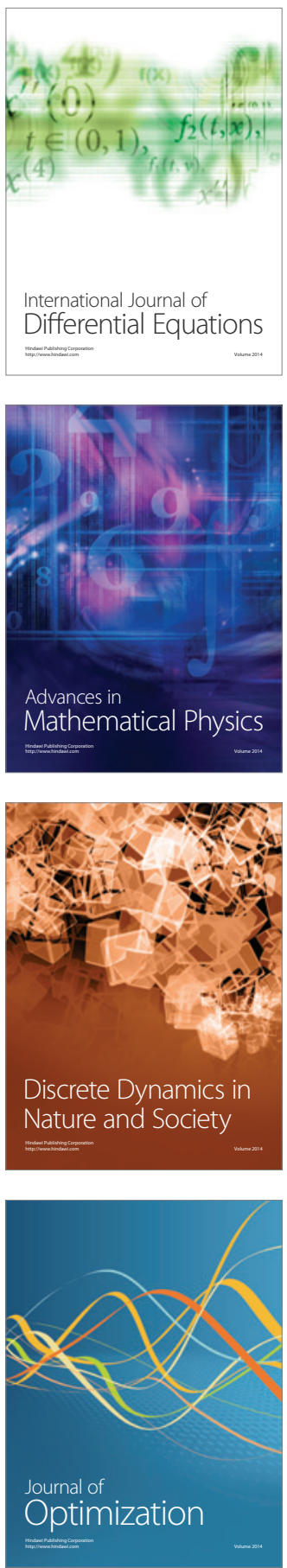